\title{
CONDUCTIVITY OF PERIODICAL STRUCTURES WITH STRONGLY DEGENERATED ELECTRON GAS
}

\author{
P. P. Petrov \\ State University "Lvivska Politekhnika" \\ 1 Kotliarevskyi Str., Lviv, UA-290013, Ukraine
}

(Received July 16, 1998; received in final form November 23, 1998)

\begin{abstract}
The conductivity of a periodical structure consisting of successive layers of a material with degenerate electron gas, divided by thin tunnel barriers, or having periodical set of planes with randomly distributed scattering centers, is calculated. In both cases a correction to resistivity increases linearly with the rise of the ratio of the bulk electron free path to the structure period. The dependence of conductivity on parameters characterizing the interfaces between conducting layers is established.
\end{abstract}

Key words: periodical structure, kinetic equation, conductivity.

PACS numbers: 72.10.-d; 73.40.Rw

The electrical resistivity of structures consisting of microregions of materials of metallic type conductivity divided by thin insulating barriers is a well known problem of the solid state physics. The interest to it has aroused due to the latest intensive investigations of metallic superlattices. As is known, the attempts of calculating the conductivity of spatial confined systems, based on the Boltzmann's equation, have had serious difficulties in the formulation of boundary conditions for the partition functions. The use of some model ideas requires introduction of phenomenological parameters in the boundary conditions. It leads to a loss of the dependence of conductivity upon such an important factor as the mean free path [1].

In this paper we consider a structure consisting of successive layers of the material with degenerate electron gas. We assume the layers to be divided from each other either by tunnel thin barriers or by some planes with randomly distributed scattering centers like impurities or interface defects. A similar situation can be realized by various ways, for example, in metallic superlattices or semiconductor structures with delta-doped layers.

The width of conducting with $z_{0}$ is assumed to be sufficiently large as compare of to the electron mean free path $\lambda$ so that the size-quantization effects in a layer could be neglected. At the same time, we assume the screening length $L$ to be small in comparison with the bulk mean free path $l_{F}=v_{F} \tau$. The above-indicated conditions can be fulfilled simultaneously for strongly degenerated electron gas (for the metallic densities of electron gas $\lambda \sim L \sim a_{o}$, where $a_{o}$ is the lattice constant).

To calculate the conductivity of the structure, we use a method based on the solution of the kinetic equation in a conducting layer with periodic boundary conditions, so that the boundary conditions at the interface are determined by the sewing up the electron wavefunctions.

Let us consider the electric current in the direction of $z$-axis, that is perpendicular to the layer plane. The kinetic equation for the partition functions inside each layer under weak electric field has the form

$$
\frac{e E(z)}{m} \frac{\partial f_{0}}{\partial v_{z}}+v_{z} \frac{\partial f}{\partial z}+\frac{f-f_{0}}{\tau}=0
$$

It accounts for the scattering from impurities in the bulk of the layer in the framework of the relaxation time $\tau$ approximation. As usual, here $f_{0}$ is the equilibrium partition function.

Let the layer $0<z<z_{0}$ be under consideration. The solution of (1) can be written separately for carriers moving along the direction of the electric field, and in the opposite direction

$$
\begin{aligned}
f^{+} & =f_{0}+C_{1}(v) e^{-z / v \tau} \\
& -e \frac{\partial f_{0}}{\partial \varepsilon} e^{-z / v \tau} \int_{0}^{z} E\left(z^{\prime}\right) e^{z^{\prime} / v \tau} d z^{\prime} \\
f^{-} & =f_{0}+C_{2}(v) e^{z / v \tau} \\
& -e \frac{\partial f_{0}}{\partial \varepsilon} e^{z / v \tau} \int_{0}^{z} E\left(z^{\prime}\right) e^{-z^{\prime} / v \tau} d z^{\prime}
\end{aligned}
$$

Here $C_{1}(v), C_{2}(v)$ are certain functions that are defined by the conditions of fitness of the wavefunctions at $z=0$ and $z=z_{0}$. Besides, in the partition functions (2) $v=v_{z}$ is assumed to be positive, i.e., we take the module of the velocity projection on the $z$-axis.

The distribution of electric field $E(z)$ in (1) and (2) differs from the constant value $E_{c}$ inside the layer only in a narrow region $\Delta$ near the interface. The boundary conditions at $z=0$ and $z=z_{0}$, as follows from (2), can be written as

$$
\begin{aligned}
& f^{+}(+0)=f_{0}+C_{1}(v), \\
& f^{-}(+0)=f_{0}+C_{2}(v),
\end{aligned}
$$




$$
\begin{aligned}
f^{+}\left(z_{0}-0\right) & =f_{0}+C_{1}(v) e^{-z_{0} / l}-e \frac{\partial f_{0}}{\partial \varepsilon} \\
& \times\left[E_{c} l\left(1-e^{-z_{0} / l}\right)+E_{0} \Delta\left(1+e^{-z_{0} / l}\right)\right], \\
f^{-}\left(z_{0}-0\right) & =f_{0}+C_{2}(v) e^{z_{0} / l}-e \frac{\partial f_{0}}{\partial \varepsilon} \\
& \times\left[-E_{c} l\left(1-e^{z_{0} / l}\right)+E_{0} \Delta\left(1+e^{z_{0} / l}\right)\right]
\end{aligned}
$$

where $l=v \tau$, and the value $E_{0} \Delta$ defines the potential drop at the interface.

The current along $z$-axis can be calculated from

$$
j_{z}(z)=2 e \int_{v_{z}>0} \frac{d^{3} k}{(2 \pi)^{3}} v_{z}\left(f^{+}-f^{-}\right)
$$

Consequently, the equation

$$
j_{z}(z)=j_{0}
$$

determines the distribution of electric fields. Here $j_{0}$ is a given value of the current flowing through the structure. Using (3), equation (5) can be presented in the form

$$
\int_{0}^{v_{F}}\left[\tilde{C}_{1}(v)-\tilde{C}_{2}(v)-2 E_{c} v \tau\right] v d v=0
$$

where new constants

$$
\begin{aligned}
& C_{1}(v)=\left(-e \frac{\partial f_{0}}{\partial \varepsilon}\right) \tilde{C}_{1}(v) \\
& C_{2}(v)=\left(-e \frac{\partial f_{0}}{\partial \varepsilon}\right) \tilde{C}_{2}(v)
\end{aligned}
$$

are introduced.

Apart from the periodicity condition of the type

$$
f^{ \pm}( \pm 0)=f^{ \pm}\left(z_{0} \pm 0\right)
$$

for the $f^{ \pm}$functions, the conditions of fitness are imposed at each interface between conducting layers.

In order to obtain the equations of fitness we follow the method proposed in [2] in the problem of scattering of electrons from the rough metal-vacuum interface, and the one developed in [3] for the problem of conductivity of the simple contact of two metals.

The carriers are quantum reflected and scattered from defects at the interface. Taking into account the elasticity of these processes, the wavefunction can be written as a superposition of states at the isoenergetic surface

$$
\begin{aligned}
\Psi(\varepsilon, \boldsymbol{\rho}) & =\int \frac{d k_{z} d^{2} k_{\rho}}{(2 \pi)^{3}} a\left(k_{z}, \mathbf{k}_{\rho}\right) e^{i\left(k_{z} z+\mathbf{k}_{\rho} \cdot \boldsymbol{\rho}\right)} \delta \\
& \times\left(\varepsilon-\frac{\left(k_{z}^{2}+k_{\rho}^{2}\right)}{2 m}\right)
\end{aligned}
$$

where $\rho=(x, y)$ is the vector perpendicular to the system axis. After evaluating the integral over $k_{z}$, we obtain an expression

$$
\begin{aligned}
\Psi_{\alpha}(\varepsilon, \boldsymbol{\rho}) & =\int \frac{d^{2} k}{(2 \pi)^{2}} \frac{1}{v^{\prime}} \\
& \times\left[a_{\alpha k}^{>} e^{i k_{z}^{\prime} z}+a_{\alpha k}^{<} e^{-i k_{z}^{\prime} z}\right] e^{i \mathbf{k} \cdot \boldsymbol{\rho}}
\end{aligned}
$$

where the denotations $\mathbf{k} \equiv \mathbf{k}_{\rho} ; k_{z}^{\prime}=\sqrt{2 m \varepsilon-k^{2}} ; v=$ $k_{z}^{\prime} / m$ are introduced (here and so on we put $\hbar=1$ ). Index $\alpha=1,2$ corresponds to the wavefunction values in the regions $0<z<z_{0}$, and $z_{0}<z<2 z_{0}$, respectively. The upper indices $(>,<)$ correspond to the two possible signs of $k_{z}^{\prime}$, therefore $a_{\alpha k}^{>}$and $a_{\alpha k}^{<}$are the amplitudes of waves moving in the opposite directions.

The Lagrangian of the system of two layers, divided by a barrier, has the form

$$
\begin{aligned}
L & =\int_{0}^{2 z_{0}} d z d^{2} \rho\left\{\frac{\left|\Psi_{1}\right|^{2}}{2 m}\left[1-\Theta\left(z-z_{0}\right)\right]\right. \\
& +\frac{\left|\Psi_{2}\right|^{2}}{2 m} \Theta\left(z-z_{0}\right)-\varepsilon\left|\Psi_{1}\right|^{2}\left[1-\Theta\left(z-z_{0}\right)\right] \\
& \left.-\varepsilon\left|\Psi_{2}\right|^{2} \Theta\left(z-z_{0}\right)+V(\boldsymbol{\rho})\left|\Psi\left(z_{0}, \boldsymbol{\rho}\right)\right|^{2} \delta\left(z-z_{0}\right)\right\} .
\end{aligned}
$$

Here $V(\boldsymbol{\rho})$ is the potential created by the distribution of impurities at the interface, $\Theta(z)$ is the step function, $\Psi=\Psi_{1}$ at $z<z_{0}, \Psi=\Psi_{2}$ at $z>z_{0}, \Psi=\Psi_{1}, \Psi_{2}$ at $z=z_{0}$. After variation in (11) over $\Psi$ we get

$$
\begin{aligned}
& -\frac{1}{2 m}\left\{\partial_{i}\left(1-\Theta\left(z-z_{0}\right)\right) \partial_{i} \Psi_{1}-\frac{1}{2 m} \partial_{i} \Theta\left(z-z_{0}\right) \partial_{i} \Psi_{2}\right. \\
& +\delta\left(z-z_{0}\right) V(\rho) \Psi-\varepsilon \Psi=0
\end{aligned}
$$

The integration of (12) within the infinitely small neighborhood $\delta \rightarrow+0$ of the point $z=z_{0}$ gives us an equation of fitness for the wavefunctions

$$
\begin{aligned}
\left.\frac{1}{2 m} \frac{d \Psi_{1}}{d z}\right|_{z=z_{0}-\delta} & -\left.\frac{1}{2 m} \frac{d \Psi_{2}}{d z}\right|_{z=z_{0}+\delta} \\
& +\left.V(\boldsymbol{\rho}) \Psi\right|_{z_{0}}=0
\end{aligned}
$$

which after substitution of (10) can be rewritten in the form 


$$
\begin{aligned}
& a_{1 k}^{>}-a_{1 k}^{<}+a_{2 k}^{<}-a_{2 k}^{>} \\
& \quad-2 i \sum_{q} \frac{V_{q}}{v^{\prime}}\left(a_{1 k-q}^{>}+a_{1 k-q}^{<}\right)=0
\end{aligned}
$$

The second equation for amplitudes is a consequence of the continuity condition for the wavefunctions at the interface $\Psi_{1}\left(z_{0}, \boldsymbol{\rho}\right)=\Psi_{2}\left(z_{0}, \boldsymbol{\rho}\right)$

$$
a_{1 k}^{>}+a_{1 k}^{<}=a_{2 k}^{>}+a_{2 k}^{<}
$$

The equations for partition functions follow from their relation with the diagonal components of the density ma- trix

$$
\left|a_{1}^{<}\right|^{2}=v f^{-}, \quad\left|a_{1}^{>}\right|^{2}=v f^{+},
$$

and the analogous formulas for the second region $z_{0}<$ $z<2 z_{0}$. The factor $v$ in these formulas is related with normalization conditions [2].

In what follows we consider two cases corresponding to the different model description of the interface between the conducting layers.

For the tunnel transparent barrier (no impurities or defects at the interface) when $V(\rho)=$ const, we get using (14) $-(16)$

$$
\left\{\begin{array}{l}
f^{-}\left(z_{0}-0\right)=R f^{+}\left(z_{0}-0\right)+(1-R) f^{-}\left(z_{0}+0\right) \\
f^{+}\left(z_{0}+0\right)=R f^{-}\left(z_{0}+0\right)+(1-R) f^{+}\left(z_{0}-0\right)
\end{array}\right.
$$

where $R(v)$ is the reflection coefficient at the barrier. The substitution in (17) of the boundary values $(3)$ of $f^{ \pm}$ accounting for their periodicity (8) and denotation (7), leads to a set of equations for determination of coefficients $\tilde{C}_{1}(v), \tilde{C}_{2}(v)$.

$$
\begin{aligned}
& R \tilde{C}_{1} e^{-z_{0} / l}+\left(1-R-e^{z_{0} / l}\right) \tilde{C}_{2}=-E_{c} l\left[\left(1-e^{z_{0} / l}\right)+R\left(1-e^{-z_{0} / l}\right)\right] \\
& +E_{0} \Delta\left[\left(1-e^{z_{0} / l}\right)+R\left(1-e^{-z_{0} / l}\right)\right], \\
& \tilde{C}_{1}\left(1-T e^{-z_{0} / l}\right)-R \tilde{C}_{2}=T E_{c} l\left(1-e^{-z_{0} / l}\right)+T E_{0} \Delta\left(1-e^{-z_{0} / l}\right) .
\end{aligned}
$$

Their solution has the form

$$
\begin{aligned}
& \tilde{C}_{1}=\frac{2(1-2 R) e^{\xi}}{\left(1-e^{\xi}\right)\left(2 R-1+e^{\xi}\right)} \chi(\xi), \\
& \tilde{C}_{2}=-\frac{2 e^{\xi}}{\left(1-e^{\xi}\right)\left(2 R-1+e^{\xi}\right)} \chi(\xi), \\
& \chi(\xi)=E_{c} l(1-\cosh (\xi))-E_{0} \Delta \sinh (\xi)
\end{aligned}
$$

where $\xi=z_{0} / l$.

Then, the equation (6) may be written

$$
\begin{aligned}
& \int_{0}^{v_{F}} v d v \frac{2(1-R) e^{\xi}}{R^{2}-\left(1-R-e^{\xi}\right)^{2}}\left[E_{c} l(1-\cosh (\xi))\right. \\
& \left.-E_{0} \Delta \sinh (\xi)\right]-\int_{0}^{v_{F}} v d v E_{c} l=0 .
\end{aligned}
$$

Counting, that reflection coefficient $R=R(v)$ is a function of electron speed, equation (20) takes the form

$$
E_{c} l \tilde{R}_{1}+E_{0} \Delta \tilde{R}_{2}-E_{0} \Delta \tilde{p}=0,
$$

where the notations

$$
\tilde{R}=\int_{0}^{v_{F}} \frac{v^{2}}{v_{F}} R(v) \varphi\left(e^{-\xi}\right) d v
$$

$$
\tilde{D}=\int_{0}^{v_{F}}(1-R(v)) \varphi\left(e^{-\xi}\right) v d v
$$

$$
\varphi(x)=-\frac{(1+x)}{1-(1-2 R) x}
$$

are used.

Adding resistance in the structure may be obtained from expression 


$$
\delta \rho=\frac{\tilde{U}}{\sigma_{0} E_{c}}
$$

where voltage drop on the barriers per length unit is determined as

$$
\tilde{U}=\frac{2 E_{0} \Delta}{z_{0}}
$$

Then, from (21), (23), and (24) follows an expression for the resistivity of structure

$$
\rho^{(1)}=\rho_{0}\left(1+\frac{2 l_{F}}{z_{0}} \frac{\tilde{R}}{\tilde{D}}\right)
$$

where $\rho_{0}$ is the bulk resistivity of the conducting layer material.

In the presence of impurities or defects at the interface we assume the corresponding random potential to be Gaussian

$$
\langle V(\rho)\rangle=0, \quad\left\langle V(\rho) V\left(\rho^{\prime}\right)\right\rangle=\gamma \delta\left(\rho-\rho^{\prime}\right),
$$

where angled brackets are for averaging over realizations of the random potential. In this case the equations of fitness at the interface has the form

$$
\begin{aligned}
& f_{k}^{-}\left(z_{0}-0\right)=f_{k}^{-}\left(z_{0}+0\right)\left(1-\frac{4 \pi \gamma}{v} \sum_{q} \frac{1}{v^{\prime}}\right) \\
& +\frac{2 \pi \gamma}{v} \sum_{q} \frac{1}{v^{\prime}} f_{k^{\prime}}^{+}\left(z_{0}-0\right)+\frac{2 \pi \gamma}{v} \sum_{q} \frac{1}{v^{\prime}} f_{k^{\prime}}^{-}\left(z_{0}+0\right), \\
& f_{k}^{+}\left(z_{0}+0\right)=f_{k}^{+}\left(z_{0}-0\right)\left(1-\frac{4 \pi \gamma}{v} \sum_{q} \frac{1}{v^{\prime}}\right) \\
& +\frac{2 \pi \gamma}{v} \sum_{q} \frac{1}{v^{\prime}} f_{k^{\prime}}^{+}\left(z_{0}-0\right)+\frac{2 \pi \gamma}{v} \sum_{q} \frac{1}{v^{\prime}} f_{k^{\prime}}^{-}\left(z_{0}+0\right)
\end{aligned}
$$

where index $k^{\prime}$ corresponds to the $z$-component of momentum, and the sum over $q$ means the summation over longitudinal components of momentum (i.e., along the interface).

Consequently, from (26) follows a new set of equations for the determination of coefficients $\tilde{C}_{1}(v), \tilde{C}_{2}(v)$

$$
\begin{aligned}
\tilde{C}_{1}\left(1-p e^{-z_{0} / l}\right) & =E_{c} l\left(1-e^{-z_{0} / l}\right) p+E_{0} \Delta\left(1+e^{-z_{0} / l}\right)+\frac{\tilde{\gamma}}{v}\left(S_{1}+S_{2}\right) \\
& +\frac{\tilde{\gamma}}{v} \int_{0}^{v_{F}} d v^{\prime}\left[E_{c} l^{\prime}\left(1-e^{-z_{0} / l^{\prime}}\right)+E_{0} \Delta\left(1-e^{-z_{0} / l^{\prime}}\right)\right] \\
\tilde{C}_{2}\left(e^{z_{0} / l}-p\right) & =E_{c} l\left(1-e^{z_{0} / l}\right)-E_{0} \Delta\left(1-e^{z_{0} / l}\right)+\frac{\tilde{\gamma}}{v}\left(S_{1}+S_{2}\right) \\
& +\frac{\tilde{\gamma}}{v} \int_{0}^{v_{F}} d v^{\prime}\left[E_{c} l^{\prime}\left(1-e^{-z_{0} / l^{\prime}}\right)+E_{0} \Delta\left(1-e^{-z_{0} / l^{\prime}}\right)\right]
\end{aligned}
$$

where

$$
\begin{aligned}
& \tilde{C}_{1}=E_{c} l+E_{0} \Delta \operatorname{cth}\left(Z_{0} / 2 l\right), \\
& \tilde{C}_{2}=-E_{c} l-E_{0} \Delta \operatorname{cth}\left(Z_{0} / 2 l\right),
\end{aligned}
$$$$
p=\left(1-2 \tilde{\gamma} \frac{v_{F}}{v}\right)
$$

$$
S_{1}=\int_{0}^{v_{F}} d v^{\prime} \tilde{C}_{1} e^{-z_{0} / l^{\prime}} ; \quad S_{2}=\int_{0}^{v_{F}} d v^{\prime} \tilde{C}_{2}
$$

and the dimensionless scattering parameter $\tilde{\gamma}=2 m^{2} \gamma$ is introduced.

In the case of weakly scattering, when the conditions $\tilde{\gamma} \ll 1$ and $p \cong 1$ are obeyed, solutions of (28) have the form

Therefore the equation (6) may be written as

$$
\begin{aligned}
& \int_{0}^{v_{F}}\left[\tilde{C}_{1}(v)-\tilde{C}_{2}(v)-2 E_{c} v l\right] v d v \\
& =\left(-2 \tilde{\gamma} E_{c} l+2 E_{0} \Delta\right) \int_{0}^{v_{F}} v d v \operatorname{cth}\left(z_{0} / 2 l\right)=0
\end{aligned}
$$


that leads to

$$
\tilde{\gamma} E_{c} l=E_{0} \Delta
$$

Making use of (33) and performing the calculations in a way similar to obtaining (25), we get an expression for the resistivity of the structure at the presence of scatters at the interface

$$
\rho^{(2)}=\rho_{0}\left(1+\frac{2 l_{F} \tilde{\gamma}}{z_{0}}\right)
$$

Note that in formula (34) we assume the parameter $\tilde{\gamma} l_{F} / z_{0} \ll 1$ to be small.

Summarizing, we conclude, that according to (25) and (34) the resistivity of structure increases linearly with parameter $2 l / z_{0}$ in both the considered cases. The difference in the character of electron scattering at the interface between the conducting layers, is taken into account with the aid of factors $\tilde{R} / \tilde{D}$ and $\tilde{\gamma}$ in these formulas.
[1] H. Sondheimer, Adv. Phys. 1, 1 (1952)

[2] L. A. Falkovsky, Adv. Phys. 32, 753 (1983)
[3] V. K. Dugaev, V. I. Litvinov, P. P. Petrov, Phys. Rev. B $\mathbf{5 2}, 5306$ (1995)

\title{
ПРОВІДНІСТЬ У ПЕРІОДИЧНИХ СТРУКТУРАХ ЗІ СИЛЬНО ВИРОДЖЕНИМ ЕЛЕКТРОННИМ ГАЗОМ
}

\author{
П. Петров \\ Державний університет "Львівсъка політехніка", \\ вул. Котляревського, 1, Львів, 290013, Украйна.
}

Обчислено провідність у періодичній структурі, яка складається з шарів провідного матеріялу, розділених тунельно прозорими бар'єрами або площинами з випадковим розподілом центрів розсіяння. В обох випадках поправка до питомого опору провідного матеріялу структури зростає лінійно зі збільшенням відношення довжини вільного пробігу електронів до періоду структури. Визначено залежність провідности структури від параметрів, шо описують ме жі розділу між провідними шарами. 